\title{
Urinary prostaglandin and prostaglandin metabolite excretion in patients with essential hypertension or hypertension with renal artery stenosis
}

\author{
C.T. Postma ${ }^{a, *}$, Th. de Boo ${ }^{\text {b }}$, C.M.G. Thomas ${ }^{\text {c }}$, Th. Thien ${ }^{a}$ \\ ${ }^{a}$ Department of Medicine, Division of General Internal Medicine, University Hospital, PO Box 9101, 6500 HB Nijmegen, Netherlands \\ ${ }^{\mathrm{b}}$ Department of Medical Statistics, University Hospital, Nijmegen, Netherlands \\ ${ }^{\mathrm{c}}$ Laboratory of Gyneacology and Obstetrics, University Hospital, Nijmegen, Netherlands
}

Received 14 February 1995; revision received 18 April 1995; accepted 31 May 1995

\begin{abstract}
Background: Recent studies have reported elevated prostaglandin levels in patients with renal artery stenosis and hypertension. To investigate whether a distinction between essential hypertension and hypertension with renal artery stenosis is possible by measuring eicosanoid excretion, we studied the excretion of these compounds in patients with essential hypertension and in hypertensives with concomitant renal artery stenosis.

Methods: The 24-h urinary excretion of metabolites of prostaglandin $\mathrm{I}_{2}$, prostaglandin $\mathrm{E}_{2}$ and metabolites of thromboxane $\mathrm{A}_{2}$ was sampled under standardised conditions, in 15 patients with essential hypertension and in 15 patients with unilateral renal artery stenosis with hypertension. Also clinical and biochemical characteristics of the subjects were analysed.

Results: The patients with renal artery stenosis had significantly lower excretion of prostaglandin $\mathrm{I}_{2}$ than did the essential hypertensive patients. However, the overlap in the values was large, thus not allowing a diagnostic differentiation according to urinary prostaglandin $I_{2}$ levels. The excretion of prostaglandin $E_{2}$ and of metabolites of thromboxane $\mathrm{A}_{2}$ showed no significant differences among the groups.

Conclusions: Measurement of urinary prostaglandin or prostaglandin metabolite excretion did not contribute to the non-invasive detection of the presence of a renal artery stenosis in the patients in this study.
\end{abstract}

Keywords: Hypertension; Renal artery stenosis; Urinary prostaglandin excretion

\section{Introduction}

Prostaglandin endoperoxides are constituents of the renal regulatory mechanism of angioten-

\footnotetext{
* Corresponding author. Tel. (080) 614782; Fax (080) 541734.
}

sin-induced hypertension, both in the animal model and in humans [1-4]. In animal studies it was shown that in renin-angiotensin-dependent hypertension the levels of plasma 6-ketoprostaglandin $\mathrm{F}_{1 \alpha}$, a stable metabolite of prostaglandin $\mathrm{I}_{2}\left(\mathrm{PGI}_{2}\right)$, and thromboxane $\mathrm{B}_{2}\left(\mathrm{PGB}_{2}\right)$, a stable metabolite of thromboxane $\mathrm{A}_{2}\left(\mathrm{PGA}_{2}\right)$, are elevated and that also urinary excretion of 6-keto- 
prostaglandin $\mathrm{F}_{1 \alpha}$ and $\mathrm{PGB}_{2}$ is increased [2]. Recent studies in humans have revealed that in subjects with renal artery stenosis, systemic plasma levels of prostaglandin $\mathrm{E}_{2}\left(\mathrm{PGE}_{2}\right)$ are higher than in patients with essential hypertension and that the renal vein plasma levels of $\mathrm{PGE}_{2}$ are higher on the stenotic side than on the non-stenotic side [3,4]. Also levels of $\mathrm{PGI}_{2}$ and of $\mathrm{PGA}_{2}$ were shown to be elevated in renal artery stenosis in humans [5-9].

The prostaglandins have a predominantly vasodilator effect at the level of the afferent glomerular arteriole $[10,11]$. The intrarenal effects of the renin-angiotensin system are mainly confined to the efferent arteriole and exert a vasoconstrictor effect through angiotensin II, whose effects are partly mediated via the vasoconstrictive activity of $\mathrm{PGA}_{2}[1,10]$. These microcirculatory changes act to preserve glomerular filtration fraction in states of impaired renal perfusion pressure $[8,13]$. If renal perfusion pressure declines, $\mathrm{PGI}_{2}$ production in the endothelial cells of the vas afferens rises [7]. The $\mathrm{PGI}_{2}$ in turn potentiates renin release by the juxtaglomerular apparatus [12,14].

Recent experiments have confirmed the importance of the mechanisms described in renal artery stenosis (RAS) in humans $[3,4,8,15,16]$. Intravenous aspirin lowered $\mathrm{PGE}_{2}$ and renin concentrations, in the human renal vein from the kidney distal to an unilateral RAS, with concomitant lowering of the blood pressure [3]. A significant decline in $\mathrm{PGE}_{2}$ and renin concentrations, after the administration of aspirin, was also reported in the renal veins distal to a unilateral RAS, in patients in whom blood pressure was cured or improved after percutaneous transluminal renal angioplasty, but not in patients in whom blood pressure did not improve after the relief of a renal artery stenosis [4]. Thus, by inhibiting $\mathrm{PGE}_{2}$ production, renin production was attenuated and blood pressure fell, at least in the acute experiments $[3,4]$.

On the basis of these experiments it can be assumed that in renovascular hypertension the renal production of $\mathrm{PGE}_{2}, \mathrm{PGI}_{2}$ and $\mathrm{PGA}_{2}$ is increased [2-4]. In essential hypertension the eicosanoids do not seem to participate directly in the control of arterial pressure and their levels have not been shown to be raised $[8,17]$. Thus, by measuring renal prostaglandin production it might be possible to differentiate between essential hypertension and hypertension with a unilateral RAS.

The least invasive and most reliable way of assessing intrarenal prostaglandin production is to measure the urinary excretion of $\mathrm{PGE}_{2}$, 6-ketoprostaglandin $\mathrm{F}_{1 \alpha}$, and $\mathrm{PGB}_{2}$ [18]. Only a slight day-to-day variation in the excretion of $\mathrm{PGE}_{2}$ has been reported [19]. We measured the 24-h urinary excretion of these eicosanoids in patients with essential hypertension and in patients with unilateral renal artery stenosis and hypertension, to examine whether the assumed differences in urinary prostaglandin excretion would be apparent.

\section{Patients and methods}

Included in the study were 15 patients with essential hypertension and 15 patients with hypertension and a unilateral renal artery stenosis. Hypertension was defined as a supine blood pressure, taken on 3 different occasions, above 90 $\mathrm{mmHg}$ diastolic on each occasion. The diagnosis of essential hypertension was made if laboratory investigation and angiography had excluded secondary forms of hypertension. Subjects with proteinuria over $1.5 \mathrm{~g}$ a day, haematuria or abnormalities on urine microscopy were excluded. Subjects with diabetes mellitus were also excluded from the study. The diagnosis of unilateral renal artery stenosis was made if on a Seldinger arteriography or on an arterial digital subtraction angiography a luminal narrowing of more than $70 \%$ of the surface was present. Fifteen successive patients were selected in whom a diagnosis of renal artery stenosis was made. The patients were included if their characteristics were in agreement with the protocol as outlined above. The subjects with essential hypertension were also 15 successive patients in whom the diagnosis of essential hypertension was made and whose clinical data matched the study terms. In all subjects 
antihypertensive medication and a sodium-restricted diet were discontinued at least 2 weeks before the urine sampling. In the setting of the outpatient department it is hardly possible to bring subjects into a fixed sodium balance. We assumed that by taking an unlimited sodium diet the patients would reach a steady state with regard to their salt status. All other medication was also withheld. Special emphasis was given to prevent the use of cyclo-oxygenase-inhibiting drugs. The patients were asked to refrain from smoking and sexual activity from $24 \mathrm{~h}$ before the start of the urine sampling.

Urine was sampled during two successive periods of $24 \mathrm{~h}$ because, in case of any collection problem, there would be a second sample. As standard the first of the two samples was used. In 2 cases the ratio of $\mathrm{PGE}_{2}$ and prostaglandin $\mathrm{F}_{2 \alpha}$, which for this purpose was also measured, was over 0.5 , indicating that the sample was contaminated by seminal fluid and therefore the second sample was used [19]. The creatinine content of the urine sample that was used for the study was measured to calculate the endogenous creatinine clearance (ECC). Twenty-four hour urinary sodium content was measured in the same 24-h urine as the prostaglandins or, if this was not feasible, it was measured in a 24 -h urine sample of the same week in which the 24-h urine for the prostaglandin measurements was taken.

The levels of $\mathrm{PGE}_{2}, \mathrm{PGF}_{2 \alpha}$, 6-keto-PGF $\mathrm{PG}_{1 \alpha}$ and $\mathrm{TxB}_{2}$ were determined by radioimmunoassays, according to procedures described previously [20,21]. After 10 consecutive assays the $\mathrm{PGE}_{2}$ determinations showed 6.4 and $15 \%$, the $\mathrm{PGF}_{2 \alpha}$ assay 5.3 and $11 \%$, the 6-keto-PGF ${ }_{1 \alpha}$ assay 7.3 and $9.6 \%$, and the $\mathrm{TxB}_{2}$ assay 8.1 and $12.5 \%$ intra- and interassay variability, respectively.

To relate prostaglandin excretion to functional renal mass, 24-h urinary prostaglandin metabolite excretion was indexed to the $24-\mathrm{h}$ creatinine content of the same sample. Plasma renin activity (PRA) was measured under baseline conditions, meaning that all antihypertensive medications were stopped for at least 2 weeks. After blood for baseline PRA was taken, patients took an oral dose of $25 \mathrm{mg}$ of captopril and $60 \mathrm{~min}$ after that blood for captopril-stimulated PRA was drawn.
During this procedure the patients remained in the supine position.

The features of gender, age, body mass index (body weight in kilograms divided by the square of the length in metres), endogenous creatinine clearance, PRA, 24-h urinary sodium content, smoking habits and blood pressure levels were compared as were the 24-h urinary $\mathrm{PGE}_{2}$, 6-keto$\mathrm{PGF}_{1 \alpha}$ and thromboxane $\mathrm{B}_{2}$ excretion and the prostaglandin levels indexed to 24 -h creatinine.

\section{Statistical analysis}

Patient characteristics among the groups were compared with Wilcoxon's rank sum test apart from the gender and the numbers of smokers, who were compared with Fisher's exact test. The 24-h urinary prostaglandin and prostaglandin metabolite excretion were also compared by Wilcoxon's rank sum test. Outcomes with a $p$ value of less than $5 \%$ were regarded as significant. All tests were performed two-sided. In a forward stepwise logistic regression procedure the independent relation of age, ECC, body mass index, smoking, systolic and diastolic blood pressure and the diagnosis of essential hypertension and renal artery stenosis to the prostaglandin and

Table 1

Clinical characteristics of patients with essential hypertension (EH) $(n=15)$ and patients with renal artery stenosis (RAS) $(n=15)$

\begin{tabular}{lll}
\hline & EH & RAS \\
\hline Gender (F/M) & $5 / 10$ & $3 / 12$ \\
Age (yrs) & $45(20-63)$ & $53(21-74)$ \\
Body mass index $\left(\mathrm{kg} / \mathrm{m}^{2}\right)$ & $25(21-33)$ & $25(21-31)$ \\
Urinary Na (mmol/24 h) & $155(40-313)$ & $154(40-200)$ \\
ECC (ml/min) & $94(64-141)$ & $82(29-124)$ \\
No. of smokers $(\%)$ & $1(6 \%)^{*}$ & $9(60 \%)$ \\
Systolic blood pressure & $170(140-240)$ & $184(144-220)$ \\
$\quad$ (mmHg) & & \\
Diastolic blood pressure & $108(80-150)$ & $110(80-130)$ \\
$\quad(m m H g)$ & & \\
\hline
\end{tabular}

Median and (between brackets) the range are indicated, unless indicated otherwise. $\mathrm{ECC}=$ endogenous creatinine clearance. ${ }^{* *}$ Indicates significant difference between $\mathrm{EH}$ and RAS, $p<0.01$. 
Table 2

Urinary prostaglandin metabolite excretion and plasma renin activity in essential hypertensives $(\mathrm{EH})(n=15)$ and patients with hypertension and renal artery stenosis $\operatorname{RAS}(n=15)$

\begin{tabular}{lll}
\hline & $\mathrm{EH}$ & $\mathrm{RAS}$ \\
\hline 6-Ketoprostaglandin $\mathrm{F}_{1 \alpha}(\mathrm{nmol} / 24 \mathrm{~h})$ & $1.9(0.54-3.6)^{* *}$ & $1.1(0.28-2.5)$ \\
Prostaglandin $\mathrm{E}_{2}(\mathrm{nmol} / 24 \mathrm{~h})$ & $0.87(0.09-13)$ & $0.8(0.23-5.3)$ \\
Thromboxane $\mathrm{B}_{2}(\mathrm{nmol} / 24 \mathrm{~h})$ & $1.2(0.3-2.1)$ & $1.2(0.42-2.7)$ \\
& & $0.08(0.04-0.27)$ \\
Prostaglandin metabolite excretion $(\mathrm{nmol} / \mathrm{l} / 24 \mathrm{~h})$ indexed to urinary creatinine excretion $(\mathrm{mmol} / \mathrm{l} / 24 \mathrm{~h})$ \\
6-Ketoprostaglandin $\mathrm{F}_{1 \alpha}$ & $0.13(0.07-0.28)^{*}$ & $0.06(0.02-0.45)$ \\
Prostaglandin $\mathrm{E}_{2}$ & $0.05(0.01-1.02)$ & $0.12(0.03-0.36)$ \\
Thromboxane $\mathrm{B}_{2}$ & $0.08(0.04-0.21)$ & \\
& & $1.9(0.4-7.7)$ \\
Plasma renin activity $(n m o l / l / h)$ & $0.87(0.1-3.96)$ & $9.8(0.1-51.1)$ \\
Baseline & $1.24(0.1-2.89)^{* *}$ & \\
After 25 mg of captopril & & \\
\hline
\end{tabular}

Given are Medians and between brackets ranges. ${ }^{*}{ }^{*}$ Indicates difference $p<0.01 ;{ }^{*}$ indicates difference $p<0.05$.

prostaglandin metabolite excretion were analysed.

\section{Results}

The study included 15 patients with essential hypertension and 15 with unilateral RAS. All patients with RAS had atherosclerotic vascular narrowing as evidenced by angiography.

The number of smokers was significantly higher in the patients with RAS (Table 1). The clinical features of gender, age and body mass index were not significantly different among the groups (Table 1). Twenty-four hour urinary sodium excretion was also not significantly different: median 155 (range $40-313$ ) $\mathrm{mmol} / 24 \mathrm{~h}$ in the $\mathrm{EH}$ group and $154(40-200) \mathrm{mmol} / 24 \mathrm{~h}$ in the group with RAS (Table 1). Blood pressure in the essential hypertensives and in those with RAS was not significantly different: $170(140-240) / 108(80-$ $150)$ and $184(144-220) / 110(80-130) \mathrm{mmHg}$, respectively (Table 1 ). Baseline PRA was 0.87 (0.1-3.96) $\mathrm{nmol} / \mathrm{l} / \mathrm{h}$ in the $\mathrm{EH}$ group and 1.9 $(0.4-7.7) \mathrm{nmol} / \mathrm{l} / \mathrm{h}$ in the RAS group, which was not significantly different (Table 2 ). The captopril-stimulated PRA was significantly higher in the patients with RAS: $9.8(0.1-51.1)$ vs. 1.24 (0.1-2.89) $\mathrm{nmol} / \mathrm{l} / \mathrm{h}$ in the $\mathrm{EH}$ group (Table 2). In the group of essential hypertensives there was no significant rise in PRA after captopril, but in the patients with a RAS the rise in PRA after captopril was significant (Table 2). Twenty-four hours 6-keto-prostaglandin $\mathrm{F}_{1 \alpha}$ excretion was with a median of $1.1(0.28-2.5) \mathrm{nmol} / 24 \mathrm{~h}$ significantly lower in the patients with RAS as compared with the value of $1.9(0.54-3.6) \mathrm{nmol} / 24 \mathrm{~h}$ in the essential hypertensives (Table 2). Also indexed to the 24-h creatinine excretion, 24-h 6-ketoprostaglandin $\mathrm{F}_{1 \alpha}$ excretion was significantly lower in the patients with RAS; $0.08(0.04-0.28)$ vs. $0.13(0.07-0.27) \mathrm{nmol} / \mathrm{mmol}$ (Table 2). Regarding the other prostaglandins and prostaglandin metabolites, no significant differences in the values for the 24-h urinary excretion among the groups were detected.

In the forward stepwise logistic procedure the diagnosis of renal artery stenosis showed a significant relationship to the 24-h 6-ketoprostaglandin $\mathrm{F}_{1 \alpha}$ urinary excretion $\left(r^{2}=0.30, p<0.01\right)$. Furthermore, $\mathrm{PGB}_{2}$ excretion showed a significant relation to smoking $\left(r^{2}=0.22, p<0.01\right)$.

\section{Discussion}

Because renovascular hypertension can be treated successfully in a large number of patients, it is worthwhile to try to identify those with this condition among the general hypertensive population [22-25]. However, the certain diagnosis of a renal artery stenosis can only be made by an- 
giography [22-25]. In view of the low prevalence of renal artery stenosis in the hypertensive population, angiography, because of its invasive nature and cost, cannot be used as a screening procedure. Therefore a sensitive and reliable non-invasive screening procedure that can detect this condition would be an important tool in clinical practice.

A number of studies have shown that in the case of stimulation of the renin-angiotensin system, especially as a result of renal ischaemia, renal eicosanoid production rises [1-9]. Since a significant renal artery stenosis is a situation of renal ischaemia, it might be expected that this condition results in an elevated renal prostaglandin production. Thus, by assessing renal eicosanoid production, which can be done reliably and non-invasively by measuring urinary prostaglandin and prostaglandin metabolite excretion, the presence of a renal artery stenosis should be detectable if the concomitant hypertension is related to the stenosis and therefore renin-angiotensin-mediated.

The patients with a RAS in the present study showed a significant rise in PRA after the administration of captopril, indicating the renin dependency of their renal haemodynamic regulation, as compared with the absence of such a rise in the essential hypertensive patients.

However, the expected higher levels of urinary prostaglandin excretion were not present in the group with RAS. On the contrary, we found a significantly lower 6-keto-prostaglandin $\mathrm{F}_{1 \alpha}$ excretion in the patients with a unilateral RAS.

How can these results be explained? One difference between the groups is the number of smokers, which was higher in the patients with RAS. To exclude as much as possible the acute effects of smoking, the participants were asked to refrain from smoking for at least $24 \mathrm{~h}$. Most of the clinical studies concerning eicosanoid production do not mention smoking habits of the studied population and in one study smoking discontinuation of $3 \mathrm{~h}$ was considered long enough to minimize its effects on eicosanoid levels [26]. Creating a group of non-smoking patients with RAS would have introduced considerable bias, as atherosclerotic renal artery disease is strongly linked to smoking. Smoking causes a rise in platelet-derived prostaglandin production, mainly of $\mathrm{TxA}_{2}$ and, probably vessel-wall-derived, prostaglandin $\mathrm{I}_{2}$ as measured by the elevated urinary excretion of their metabolites $\mathrm{TxB}_{2}$ and 6-ketoprostaglandin $F_{1 \alpha}[27,28]$. We could not substantiate such a rise because the levels of $\mathrm{TxB}_{2}$ were comparable in the two groups, although $\mathrm{TxB}_{2}$ excretion showed a relation to smoking. The level of $\mathrm{PGI}_{2}$ was lower in the group of RAS with the highest number of smokers. Because smoking would have enhanced the differences expected, it seems very unlikely that the difference in smoking could have substantially influenced the results of prostaglandin $\mathrm{I}_{2}$ and of 6-ketoprostaglandin $\mathrm{F}_{1 \alpha}$ excretion. Apart from $\mathrm{TxB} 2$, the other prostanoid excretions also did not show a relation to smoking.

Other causes of diminished 6-ketoprostaglandin $F_{1 \alpha}$ excretion might be found in a difference in functional renal mass or the presence of parenchymal renal disease. A severely diminished renal mass can cause a decline in renal prostaglandin production because renal tissue produces the renal prostanoids. Renal parenchymal diseases can give rise to various changes in renal prostaglandin production including an increase or decrease of the excretion depending on the offending condition [29-32]. However, between the two groups the endogenous creatinine clearance was not statistically different, indicating a comparable renal functional mass, and also the eicosanoid excretion indexed to creatinine excretion showed a lower 6-ketoprostaglandin $F_{1 \alpha}$ excretion in the patients with a RAS, which makes a diminished functional renal mass unlikely as the cause of the different findings. As we excluded patients with parenchymal renal disease, the difference in eicosanoid excretion can also not be explained by possible renal diseases.

What other explanation for the results of the present study might be valid? In a recent study of renal vein prostaglandin concentrations before and after cyclo-oxygenase inhibition with aspirin DL-lysine it was found that the level of prostaglandins in the veins of the stenotic kidney was increased in relation to the other kidney and declined within 30 mins after intravenous aspirin 
DL-lysine [3,4]. Although the level of the prostaglandins in the aorta was also higher than in patients with essential hypertension and low renin, they were not lower than in essential hypertensive patients with high renin, and as renal flow was not measured it remains unclear if renal prostaglandin production was in fact increased. If the concentration changes were caused by changes in flow, it is clear that such changes cannot be detected by differences in 24-hour urinary eicosanoid excretion.

As there were no significant differences in baseline PRA between our patients with essential hypertension and those with RAS, it might well be that the only differences present in such patients are differences in prostaglandin concentrations between the affected and non-affected renal veins.

To elucidate this problem, both hormone concentrations and renal blood flow have to be studied. Probably then an explanation can be given for the absence of the expected differences in prostaglandin excretion in the present study.

Due to the overlap in individual values, 6-ketoprostaglandin $F_{1 \alpha}$ excretion could also not be used to make a diagnostic differentiation between the subjects. Thus, by measuring the concentrations of renal prostaglandin or prostaglandin metabolites in the urine, no distinction could be made between essential hypertension and hypertension with renal artery stenosis.

\section{References}

[1] Wilcox CS, Welch WJ. Angiotensin II and thromboxane in the regulation of blood pressure and renal function. Kidney Int 1990; 38(suppl 30):S81-83.

[2] Lin L, Mistry M, Stier CT Jr, Nasjletti A. Role of prostanoids in renin-dependent and renin independent hypertension. Hypertension 1991; 17:517-525.

[3] Imanishi M, Kawamura M, Akabane S, et al. Aspirin lowers blood pressure in patients with renovascular hypertension. Hypertension 1989; 14:461-468.

[4] Imanishi M, Kawamura M, Akabane S, et al. The utility of aspirin for differential diagnosis of renovascular hypertension from hyperreninemic essential hypertension. AJH $1991 ; 4: 761-768$.

[5] Blackshear JL, Spielman WS, Knox FG, Romero JC. Dissociation of renin release and renal vasodilation by prostaglandin synthesis inhibitors. Am J Physiol 1979; 237:F20-F24.

[6] Jackson EK, Gerkens JF, Brash AR, Branch RA. Acute renal artery constriction increases renal prostaglandin $I_{2}$ biosynthesis and renin release in the dog. $\mathrm{J}$ Pharmacol Exp Ther 1982; 222:410-413.

[7] Romero JC, Knox FG. Mechanisms underlying pressurerelated natriuresis: the role of the renin-angiotensin and prostaglandin systems. Hypertension 1988; 11:724-738.

[8] Romero JC, Strong CG. The effect of indomethacin blockade of prostaglandin synthesis on blood pressure of normal rabbits and rabbits with renovascular hypertension. Circ Res 1977; 40:35-41.

[9] McGiff JC. Interactions of prostaglandins with the kallikrein-kinin and renin-angiotensin systems. Clin Sci 1980; 59:105s-116s.

[10] Edwards RM. Segmental effects of norepinephrine and angiotensin II on isolated renal microvessels. Am J Physiol 1983; 244:F526.

[11] Edwards RM. Effects of prostaglandins on vasoconstrictor action in isolated renal arterioles. Am J Physiol 1985; 248:F779.

[12] Olivari MT, Levine TB, Cohn JN. Evidence for a direct renal stimulating effect of prostaglandin $E_{2}$ on renin release in patients with congestive heart failure. Circulation $1986 ; 74: 1203-1207$.

[13] Packer M. Interaction of prostaglandins and angiotensin II in the modulation of renal function in congestive heart failure. Circulation 1988; 77(suppl I):I-64.

[14] Beierwaltes WH, Schrijver S, Sanders E, Strand J, Romero JC. Renin release selectively stimulated by prostaglandin $\mathrm{I}_{2}$ in the isolated rat glomeruli. Am J Physiol 1982; 243:F276-F283.

[15] Levenson DJ, Dzau VJ. The effects of angiotensin converting enzyme inhibition in renal artery stenosis. Kidney Int 1987; 31:173-179.

[16] Johnston CI, Jackson B. Overview: angiotensin converting enzyme inhibition in renovascular hypertension. Kidney Int $1987 ; 31: 154-156$.

[17] Terragno NA, Terragno DA, McGiff JC. Contribution of prostaglandins to the renal circulation in conscious, anesthesized, and laparotomized dogs. Circ Res 1977; 40:590-595.

[18] Frohlich JC, Wilson TW, Sweetman BJ et al. Urinary prostaglandins: identification and origin. J Clin Invest $1975 ; 55: 763-772$.

[19] Koopmans PP, Thomas ChMG, van de Berg RJ, Thien Th. The urinary excretion of prostaglandins and thromboxane $B_{2}$ in healthy volunteers: a study in males and females, and on the influence of seminal fluid contamination. Prostaglandins Leukotrienes Essential Fatty Acids 1988; 32:107-111.

[20] Thomas CMG, van de Berg RJ, de Koning-Gans HJ, Lequin RM. Radio-immunoassays for prostaglandins: technical validation of prostaglandin $F_{2 \alpha}$ measurements in human plasma using Sephadex G-25 gel filtration. Prostaglandins 1978; 15:839-847. 
[21] Thomas CMG, van de Berg RJ, de Koning-Gans HJ, Lequin RM. Radio-immunoassays for prostaglandins: measurement of prostaglandin $E_{2}$ and the 13,14-dihydro15-keto metabolites of the $E$ and $F$ series. Description of a reliable technique with an universal applicability. Prostaglandins 1978; 15:849-855.

[22] Postma CT, van der Steen PHM, Hoefnagels WHL, de Boo Th, Thien Th. The captopril test in the detection of renovascular disease in hypertensive patients. Arch Intern Med 1990; 150:625-628.

[23] Svetkey LP, Himmelstein SI, Dunnick NR, et al. Prospective analysis of strategies for diagnosing renovascular hypertension. Hypertension 1989; 14:247-257.

[24] Postma CT, van Oijen A, Barentsz JO, de Boo Th, Hoefnagels WHL, Thien Th. The value of tests predicting renovascular hypertension in patients with renal artery stenosis treated by angioplasty. Arch Intern Med 1991; 151:1531-1535.

[25] Mann SJ, Pickering TG, Sos TA, et al. Captopril renography in the diagnosis of renal artery stenosis: accuracy and limitations. Am J Med 1991; 90:30-40.

[26] Fitzgerald GA, Smith B, Pedersen AK, Brash AR. Increased prostacyclin biosynthesis in patients with severe atherosclerosis and platelet activation. $\mathrm{N}$ Engl J Med 1984; 3410:1065-1068.
[27] Nowak J, Murray JJ, Oates JA, FitzGerald GA. Biochemical evidence of a chronic abnormality in platelet and vascular function in healthy individuals who smoke cigarettes. Circulation 1987; 76:6-14.

[28] Chiabrando C, Rivoltella L, Alberti E, Bonollo M, Djurup R, Fanelli R. Urinary excretion and origin of 11-dehydro-2,3-dinor-thromboxane $\mathrm{B}_{2}$ in man. Prostaglandins 1993; 45:401-411.

[29] Oates JA, FitzGerald GA, Branch RA, Jackson EK, Knapp HR, Jackson Roberts L. Clinical implications of prostaglandin and thromboxane formation. Part I. N Engl $J$ Med 1988; 319:689-698.

[30] Pierucci A, Simonetti BM, Pecci G, et al. Improvement of renal function with selective thromboxane antagonism in lupus nephritis. N Engl J Med 1989; 320:421-425.

[31] Eriksson L-A, Sturfelt G, Thysell H, Wollheim FA. Effects of sulindac and naproxen on prostglandin excretion in patients with impaired renal function and rheumatoid arthritis. Am J Med 1990; 89:313-321.

[32] Mizuiri S, Takano M, Hayashi I, Aikawa A, Ohara T, Hasegawa A. Effect of prostaglandins on renal function in uninephrectomized humans. Nephron 1993; 63:429433. 